DOI: $10.5380 /$ rmu.v2i1.40678

\title{
DIA MUNDIAL DO RIM
}

O "World Kidney Day" surgiu em 2006, graças a uma iniciativa conjunta da "International Society of Nephrology" (ISN) e da "International Federation of Kidney Foundations" (IFKF).

O "World Kidney Day" procura conscientizar a todos sobre a importância dos rins para a saúde geral e reduzir a frequência e impacto da doença renal e problemas de saúde a ela associados em todo o mundo.

O "World Kidney Day" tem sido um guia para nefrologistas, suas sociedades e associações, em todo o globo, fornecendo uma série de orientações aos interessados em seu próprio "site" (http://www.worldkidneyday.com) - o que fazer, que aspectos enfatizar, que atividades desenvolver- e disponibilizando informativos e modelos de materiais, que podem ser integralmente reproduzidos, com a manutenção da sua marca.

A cada ano um tema principal é lançado, como descrito na tabela que se segue, e retratam os alvos dessa iniciativa.

Tabela1: Temáticas propostas a cada ano pelo "World Kidney Day" desde a sua criação.

\begin{tabular}{ll}
\hline Ano & Temas originais \\
\hline $\mathbf{2 0 0 6}$ & Are your kidneys OK? \\
$\mathbf{2 0 0 7}$ & CKD: common, harmful and treatable \\
$\mathbf{2 0 0 8}$ & Your amazing kidneys! \\
$\mathbf{2 0 0 9}$ & Protect your kidneys: Keep your pressure down \\
$\mathbf{2 0 1 0}$ & Protect your kidneys: Control diabetes \\
\hline $\mathbf{2 0 1 1}$ & Protect your kidneys: Save your heart \\
\hline $\mathbf{2 0 1 2}$ & Donate - Kidneys for Life - Receive \\
\hline $\mathbf{2 0 1 3}$ & Kidneys for Life - Stop Kidney Attack! \\
\hline $\mathbf{2 0 1 4}$ & Chronic Kidney Disease (CKD) and Aging \\
\hline $\mathbf{2 0 1 5}$ & Kidney Heath for All \\
\hline
\end{tabular}




\section{CAMPANHA PREVINA-SE}

Face ao aumento alarmante na prevalência da doença renal crônica (DRC) nos últimos anos e ao subdiagnóstico da doença, a Diretoria da SBN (gestão 2003-2004) sob a presidência do Dr. João Egídio Romão Jr. criou um grupo para trabalhar com a prevenção de DRC no Brasil, sob a coordenação da Dra. Gianna Mastroianni Kirsztajn, também integrante desta Diretoria. Assim, surgiu a "Campanha Previna-se" em 2003, três anos antes da iniciativa internacional "World Kidney Day"(Dia Mundial do Rim).

A "Campanha Previna-se" foi desde o seu início uma campanha contínua de prevenção de doenças renais, que ocorria ao longo de todo o ano e não apenas em datas festivas. Representava um chamado a prevenir-se de doenças renais e dirigia-se com enfoques diferentes a todos (médicos, população e autoridades).

Encontram-se entre os objetivos principais da "Campanha Previna-se": (1) divulgar o que é doença renal crônica (DRC) e como prevenir a doença; (2) alertar e conscientizar a população, autoridades e profissionais do setor de saúde sobre o problema; (3) fazer campanhas para deteç̧ão da doença em fases iniciais; (4) identificar indivíduos de maior risco de progredir para insuficiência renal; (5) alertar os nefrologistas e médicos sem especialização em Nefrologia para realizar diagnóstico e intervenções precoces para prevenir a progressão da DRC. (Adaptado de Mastroianni Kirsztajn, G. \& Souza, E. Retrospectiva da prevenção da doença renal no Brasil e no mundo: "Campanha Previnase" e "World Kidney Day". In: Atualidades em Nefrologia 13. São Paulo, Sarvier, 2014.)

\section{Gianna Mastroianni Kirsztajn}

Chefe da Disciplina de Nefrologia da UNIFESP Diretora do Departamento de Prevenção de Doenças Renais da Sociedade Brasileira de Nefrologia 